\title{
Central pathway of photic reflex myoclonus
}

\author{
Tadashi Kanouchi, Takanori Yokota, Tomoyuki Kamata, Kenji Ishii, Michio Senda
}

\begin{abstract}
Direct, new evidence for the cortical origin of photic reflex myoclonus in a patient with "posterior cortical atrophy" is provided. Photic stimulation elicited myoclonic jerks in the right upper limb muscles. An $\mathrm{H}_{2}{ }^{15} \mathrm{O}-\mathrm{PET}$ activation study with photic stimulation showed increased regional cerebral blood flow not only in both striate cortices but in the left premotor and primary motor areas as well. Transcranial magnetic stimulation over the area of the left occipital cortex elicited motor evoked potentials in the right upper limb muscles. It is concluded that in the central pathway of photic reflex myoclonus the contralateral occipital cortex is activated first, then the impulses propagate intrahemispherically to the primary motor cortex, to elicit myoclonic jerks.
\end{abstract}

(F Neurol Neurosurg Psychiatry 1997;62:414-417)

Keywords: photic reflex myoclonus; $\mathrm{H}_{2}{ }^{15} \mathrm{O}-\mathrm{PET}$; transcranial magnetic stimulation

Photic reflex myoclonus is a stimulus sensitive myoclonus induced at a fixed latency by photic stimulation. This phenomenon is an important cue for elucidating how visual inputs influence the motor system. Animal studies have indicated a cortical origin of photic reflex myoclonus. ${ }^{1-3}$ Experiments in the photosensitive baboon, Papio papio, show that the motor and occipital cortices have important roles in photoinduced myoclonus and seizure. $^{4-7}$ We here report new evidence for the cortical origin of photic reflex myoclonus in humans, with PET and transcranial magnetic stimulation (TMS).

Positron Medical

Metropolitan Institute of Gerontology, Tokyo,

Japan

K Ishii

M Senda

Correspondence to :

Dr Takanori Yokota,

Department of Neurology,

Tokyo Medical and Dental

University, 1-5-45, Yushima,

Bunkyo-ku, Tokyo 113,

Japan.

Received 12 April 1996

and in revised form

3 July 1996

Accepted 16 July 1996 limb and neck muscles. No myoclonic jerks were seen on the left side. Although the visual acuities were normal, the visual field was constricted, especially on the right side. She also showed a Balint's syndrome, cortical sensory loss in the right upper limb, and a dressing apraxia. Intellect and memory were almost normal. Brain MRI showed slight ventricular enlargement in the posterior horn and moderate cortical atrophy, most pronounced in parieto-occipital areas especially on the left side. The clinical picture of her illness was similar to the "posterior cortical atrophy" reported by Benson et al, ${ }^{8}$ but we could not make a precise diagnosis.

Somatosensory evoked cortical potentials (P25-N32) were enlarged with normal latencies by the right median nerve stimulation $(13.3 \mu \mathrm{V})$, but not by the left one $(2 \cdot 8 \mu \mathrm{V})$. By electrical stimulation of the right median nerve at the wrist, extremely increased long loop reflexes were elicited in the relaxed right flexor carpi radialis muscle at a latency of about $38 \mathrm{~ms}$.

\section{Methods}

We examined myoclonic jerks in the right flexor carpi radialis muscle during $1 \mathrm{~Hz}$ flash stimulation to full visual field using a goggle type, light emitting diode stimulator (SES107, NEC Sanei, Tokyo). Visual evoked potentials (VEPs) to the same stimulation were studied from 20 scalp electrodes (the international 10-20 system) referred to the ear lobe ipsilateral to the exploring electrodes, using a band pass filter of 3 to $3000 \mathrm{~Hz}$. Each VEP study was repeated twice after averaging more than a 50 responses.

An $\mathrm{H}_{2}{ }^{15} \mathrm{O}$-PET activation study also was done with $1 \mathrm{~Hz}$ flash stimulation described above and at eye closed rest. The regional cerebral blood flow was measured repeatedly for two tasks by the PET autoradiographic method with a bolus injection of $1.5 \mathrm{GBq}_{2}{ }^{15} \mathrm{O}$ followed immediately by two minutes of scanning with a PET camera HEADTOME-IV (Shimadzu, Kyoto). Details of the data acquisition and analysis methods have been described elsewhere. ${ }^{9}$ The normalised and subtracted cerebral blood flow images were registered to the MRIs by the 3D shift and rotation for anatomical reference, ${ }^{10}$ and change in the regional cerebral blood flow was evaluated. 
Figure 1 Rectified and averaged photic reflex myoclonus recorded in the right flexor carpi radialis muscle and VEPs to $1 \mathrm{~Hz}$ flash stimulation from the representative scalp electrodes. Abnormally large VEPs are seen especially from the electrodes over the left frontocentral area. The onset latency of abnormally large potential at the $C 3$ (arrowhead) is $48 \cdot 3 \mathrm{~ms}$. The onset latency of photic reflex myoclonus (arrow) is $62.2 \mathrm{~ms}$. The time interval between them $(13.9 \mathrm{~ms})$ is almost equal to the latency of $M E P$ elicited by the left hand motor area stimulation $(13.8 \mathrm{~ms})$.

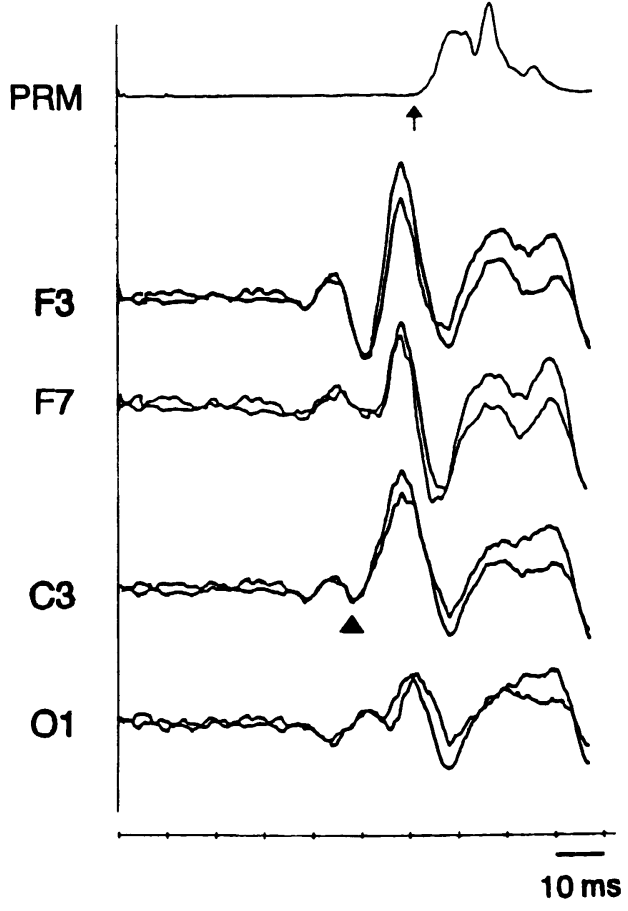

TMS over the left hand motor and occipital areas was done with a Magstim 200 (the Magstim Company, Witland, Dyfed) through a figure of eight coil. Motor evoked potentials

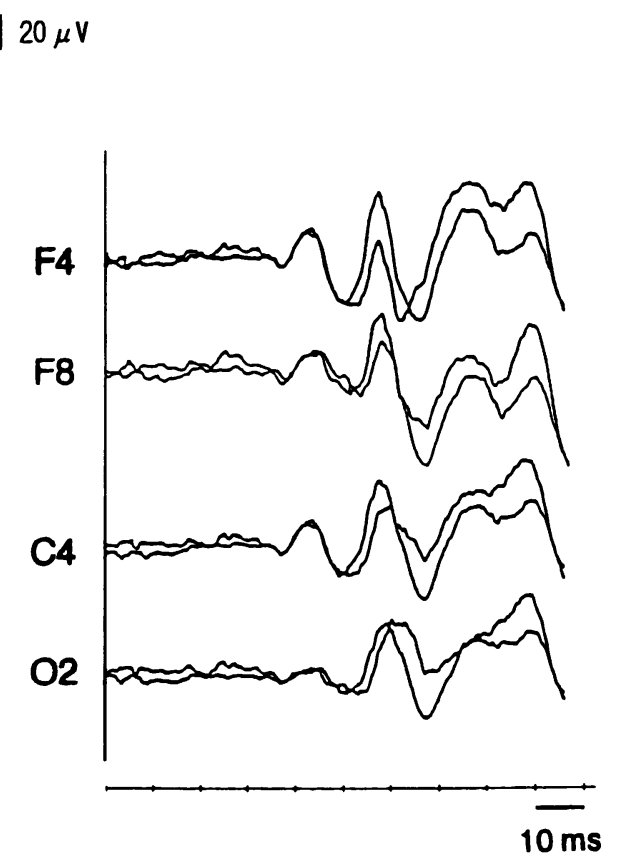

$25 \mu V$

(MEPs) of the right flexor carpi radialis muscle and the topographic map of their cortical representation over the occipital region were studied by TMS at the intensity of the stimula-

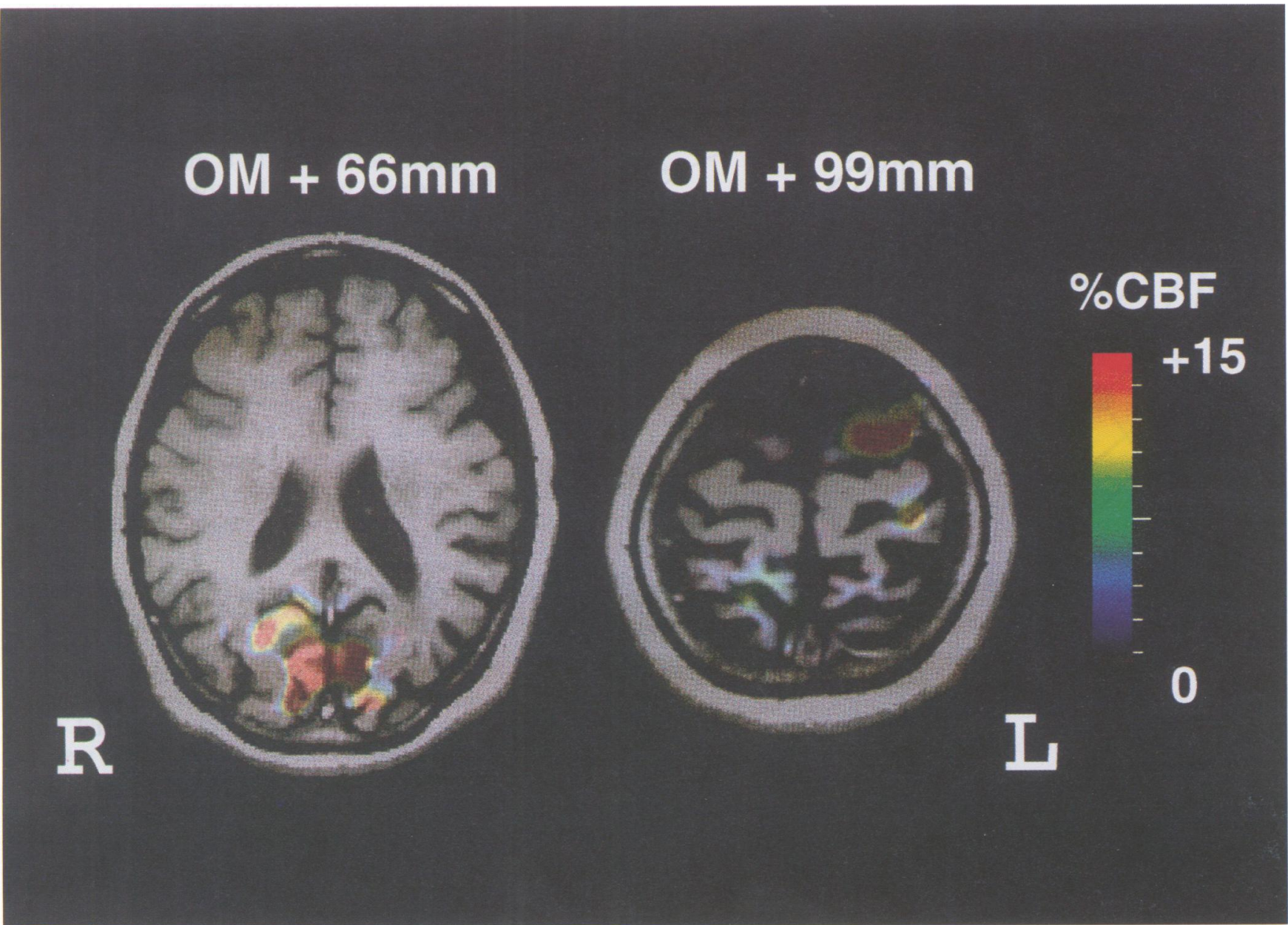

Figure 2 Subtracted PET images (1 Hz flash minus rest) are superimposed on the MRI. The increased regional cerebral blood flow is shown not only in both the striate cortices but in the left premotor and primary motor cortex. Note that the cortical atrophy is more severe in the left occipital cortex than the right. 
tor's maximal output and in the optimal direction for eliciting the largest MEPs. Stimulus sites were determined using a modified latitude/longitude based coordinate system ${ }^{11}$ :
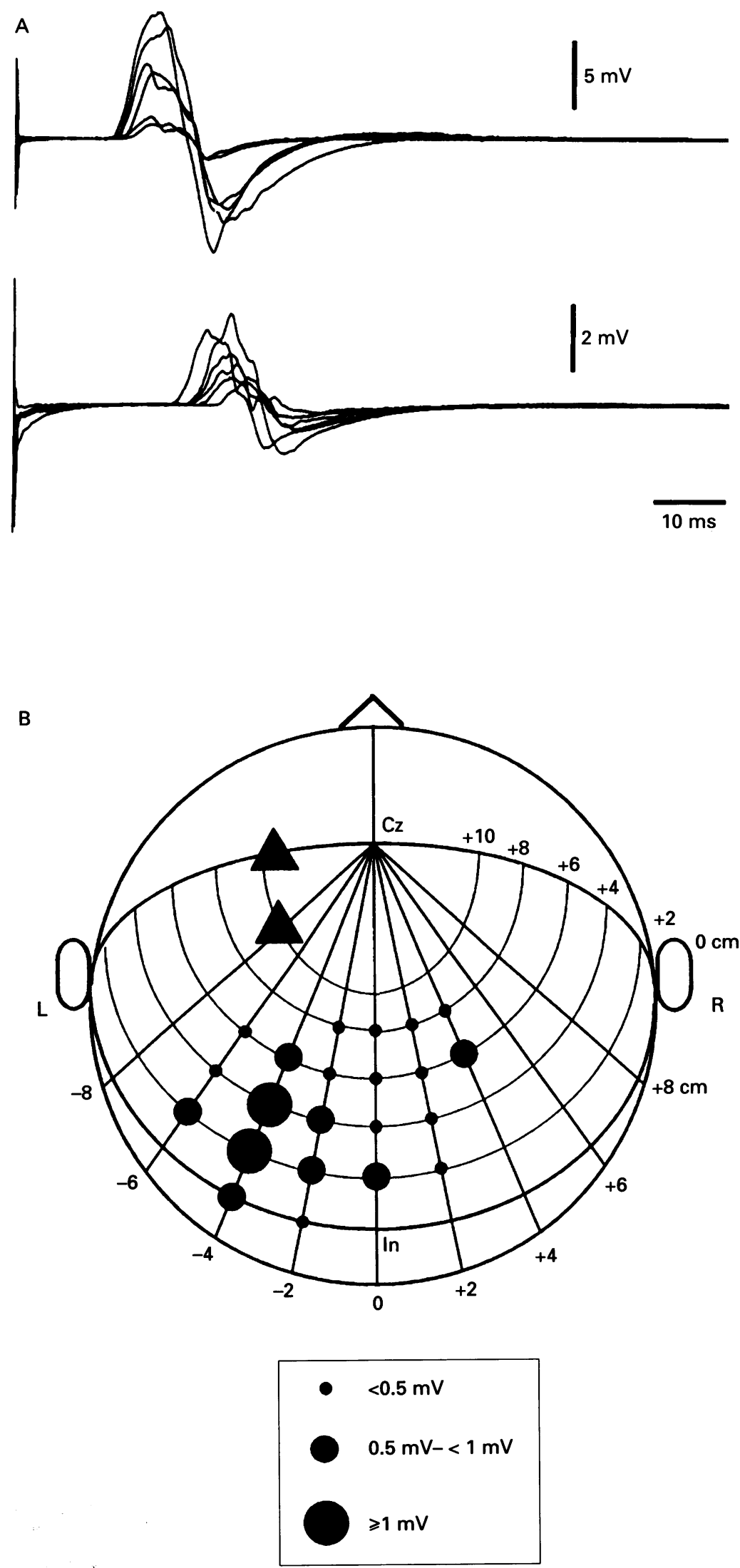

Figure 3 "(A) Representative MEPs in the right flexor carpi radialis muscle elicited by TMS over the left hand motor area (upper) and left occipital region (lower). (B) Topographic map of the scalp for MEPs in the right flexor carpi radialis muscle. Closed circles represent the sites at which TMS elicited MEPs. Circle sizes represent the mean amplitudes of four to eight MEPs. Closed triangles = the left hand motor area; In = inion.
Latitude lines $2 \mathrm{~cm}$ apart were set parallel to the reference line passing through the inion and the external acoustic meatuses. Longitude lines joined $\mathrm{Cz}$ (the 10-20 system) with each point, segmenting the reference line at intervals of $2 \mathrm{~cm}$. Successive positions were stimulated until the area in which the MEPs were elicited was surrounded by inactive positions. TMS over the occipital region also was done at rest and under voluntary contraction of the flexor carpi radialis muscles in six healthy volunteers (age 21-36).

\section{Results}

A $1 \mathrm{~Hz}$ flash stimulation induced myoclonic jerk at latencies from 61.2 to $72.4 \mathrm{~ms}$. The latency of the rectified and averaged myoclonic response was $62 \cdot 2 \mathrm{~ms}$ (fig 1 ).

VEPs to $1 \mathrm{~Hz}$ full field flash stimulation were of abnormally large size especially over the left scalp (fig 1). At the C3 electrode the amplitude of abnormally large negativepositive potential at the onset latency of $48 \cdot 3 \mathrm{~ms}$ was $28 \cdot 3 \mu \mathrm{V}$. The corresponding one at the $\mathrm{C} 4$ electrode was $19 \cdot 7 \mu \mathrm{V}$.

We carefully watched beside the patient during the PET study, but no myoclonic jerks were visible at all, even at $1 \mathrm{~Hz}$ flash stimulation which usually elicited mycclonus. The flash stimulation increased the regional cerebral blood flow more than $20 \%$ in both the bilateral striate cortices and the left premotor and primary motor areas (fig 2).

TMS over the left hand motor area elicited MEPs in the relaxed right flexor carpi radialis muscle with latencies from 13.2 to $14.8 \mathrm{~ms}$ (fig 3A (upper)). The latency of rectified and averaged MEP was $13.8 \mathrm{~ms}$. TMS over the left occipital region also produced MEPs with latencies from 21.7 to $30.3 \mathrm{~ms}$ (fig $3 \mathrm{~A}$ (lower)). The optimal direction of TMS was diagonal to the reference line. The topographic map shows that the sites at which MEPs were elicited were distributed over an area that closely corresponded to the left occipital cortex and clearly separated from the left hand motor area (fig 3B). No MEPs were elicited in either flexor carpi radialis muscle in six healthy volunteers by TMS over the occipital region.

\section{Discussion}

We provide two new lines of evidence for the cortical origin of photic reflex myoclonus given by the PET activation study and the TMS study. We also show which side of the cortex is responsible for the myoclonus.

The responsibility of the left frontal cortex contralateral to the side of myoclonic response is suggested by the $\mathrm{H}_{2}{ }^{15} \mathrm{O}$-PET activation study. Because no involuntary movements were seen during the PET study, even with 1 $\mathrm{Hz}$ flash stimulation, the increased regional cerebral blood flow in the left primary sensorimotor area was not a result of secondary activation of this cortex by muscle afferents due to myoclonic jerks. We consider this result to be subliminal cortical activation that causes 
photic reflex myoclonus, because $1 \mathrm{~Hz}$ flash stimulation usually evoked myoclonic jerks. The lateralised increase of regional cerebral blood flow is consistent with the lateralised clinical symptom. Based on the results of VEPs to flash stimulation and their cortical topographic mapping in six patients with photic reflex myoclonus, Artieda and Obeso suggested that the abnormal activity in the premotor cortex gives rise to the myoclonic jerks, but they could not define whether this abnormal cortical discharge elicits myoclonic jerks through the direct descending pathway arising in the premotor cortex or via the primary motor cortex. ${ }^{12}$ Our PET study clearly shows that the primary motor cortex as well as the premotor cortex is associated with photic reflex myoclonus.

The lateralised result of VEPs might reflect the lateralised cortical hyperexcitability as the finding of somatosensory evoked cortical potentials showed. The distribution of larger VEPs is consistent with the result of PET studies. The time interval between the onset of abnormally large VEP from the $\mathrm{C} 3$ electrode $(48.3 \mathrm{~ms})$ and the onset of myoclonic jerks $(62.2 \mathrm{~ms})$ was $13.9 \mathrm{~ms}$, the value almost equal to the latency of MEP elicited by stimulation of the left motor area $(13.8 \mathrm{~ms})$. It is suggested that the left primary motor cortical activity ultimately elicits a myoclonic jerk through the fast corticospinal pathway.

The TMS investigation showed the efferent pathway from the occipital cortex through which the contralateral muscle response is elicited. In one patient with photic reflex myoclonus, Artieda and Obeso showed that TMS given over the occipital region elicited muscle responses during steady state flash stimulation $^{12}$; but they did not identify the precise stimulated site. We first showed topographically that the area over which TMS elicits MEPs corresponds to the occipital cortex contralateral to the side of myoclonic response. The latencies are very similar to that shown by Artieda and Obeso. ${ }^{12}$ The MEPs elicited by right occipital stimulation are explained by current spread to the left occipital cortex because TMS was given in a diagonal direction at high intensity.

We therefore hypothesise that there is a central pathway of photic reflex myoclonus: A flash stimulus first activates the occipital cortex, after which impulses pass to the ipsilateral primary motor cortex, finally eliciting a contralateral myoclonic jerk through the corticospinal tract. Although our data do not exclude the cortico-subcorticocortical pathway from the occipital cortex to the frontal cortex, ${ }^{1}$ we consider it to be likely that the impulse for eliciting myoclonic response conducts the intrahemispheric occipitofrontal projections. The anatomical data for primates show that the projections from the visual area terminate in the premotor area and a part of them in the precentral area. ${ }^{513}$ It is suggested that these intrahemispheric occipitofrontal projections play a part in visual guidance of relatively independent hand and finger movements in rhesus monkeys. ${ }^{14}$ In photosensitive baboons, Papio papio, a current source density study showed that the frontorolandic paroxysmal discharges, which preceded the photo induced myoclonus, were generated mainly in layer III in the primary motor cortex probably by the arrival of visual corticocortical afferents. ${ }^{6}$

Which cortices primarily cause photic reflex myoclonus? In the patients reported by Artieda and Obeso, abnormally large VEPs indicating tangential dipole were evoked in the premotor area, while VEPs over the occipital region were normal..$^{12}$ In three patients with photic reflex myoclonus, Shibasaki and Neshige showed that extremely enhanced VEPs were evoked in the occipital region as well as the central region. ${ }^{15}$ Based on their investigation of recovery of evoked cortical responses and of myoclonic jerks, they considered that hyperexcitability of the occipital cortex may not be prerequisite to that of the motor cortex. In our patient, MRI clearly showed that the cortical atrophy was most prominent in the occipital region, especially on the left side, which is the contralateral to photic reflex myoclonus. Therefore, we speculate that not only the frontal but the occipital cortex functions in the generation of photic reflex myoclonus.

We are grateful to Dr Hiroshi Shibasaki, Department of Brain Pathophysiology, Kyoto University School of Medicine, for his helpful suggestions. Special thanks to Dr Tetsuo Futukawa, Department of Neurology, Tokyo Medical and Dental University, for his help.

1 Hunter J, Ingvar DH. Pathways mediating metrazol induced irradiation of visual impulses. Electroencephalogr Clin Neurophysiol 1955; 7:39-60.

2 Lorentz de Haas AM, Lombroso C, Merlis JK. Participation of the cortex in experimental reflex myoclonus. Electroencephalogr Clin Neurophysiol 1953;5: myoclon $177-86$.

3 Wall PD, Rémond AG, Dobson RL. Studies on the mechanism of the action of visual afferents on motor cortex excitability. Electroencephalogr Clin Neurophysiol 1953;5: 385-93.

4 Brailowsky S, Silva-Barrat C, Ménini Ch., Riche D, Naquet R. Effects of localized, chronic GABA infusions into different cortical areas of the photosensitive baboon, Papio papio. Electroencephalogr Clin Neurophysiol 1989;72: 147-56.

5 Catier J, Charmasson G, Christolomme A. Study of ipsilateral cortico-cortical connections from the occipital lobe in the photosensitive baboon. I Physiol Paris 1973;66: 93-100.

6 Silva-Barrat C, Brailowsky S, Levesque G, Ménini Ch. Epileptic discharges induced by intermittent light stimulation in photosensitive baboons: a current source density study. Epilepsy Res 1988;2:1-8.

7 Wada JA, Catier J, Charnasson G, Menini C, Naquet R. Further examination of neural mechanisms underlying photosensitivity in the epileptic Senegalese baboon, photosensitivity in the epileptic Senegalese baboon,
Papio papio. Electroencephalogr Clin Neurophysiol 1973;34: 786.

8 Benson DF, Davis RJ, Snyder BD. Posterior cortical atrophy. Arch Neurol 1988;45:789-93.

9 Senda M, Kanno I, Yonekura Y, et al. Comparison of anatomical standardization methods regarding the sensorimotor foci localization and between-subject variation in $\mathrm{H}_{2}{ }^{15} \mathrm{O}$ PET activation, a three-center collaboration study. Ann Nucl Med 1994;8:201-7.

10 Senda $M$. Mapping cortical functions using PET activation technique. In: Sugishita $\mathrm{M}$, ed. New horizons in neuropsychology. Amsterdam: Elsevier, 1994:23-34.

11 Wilson SA, Thickbroom GW, Mastaglia FL. Transcranial magnetic stimulation mapping of the motor cortex in magnetic stimulation mapping of the motor

12 Artieda J, Obeso JA. The pathophysiology and pharmacology of photic cortical reflex myoclonus. Ann Neurol 1993;34:175-84.

13 Pandya DN, Kuypers HGJM. Cortico-cortical connections in the rhesus monkey. Brain Res 1969;13:13-36.

14 Haaxma R, Kuypers HGJM. Intrahemispheric cortical connexions and visual guidance of hand and finger movements. Brain 1975;98:239-60.

15 Shibasaki $H$, Neshige $R$. Photic cortical reflex myoclonus. Ann Neurol 1987;22:252-7. 